\title{
Study of strain-induced structural, electronic, mechanical and transport properties of one-dimensional monoatomic ultrathin gold nanowire : A DFT-NEGF approach
}

\author{
Shambhu Bhandari Sharma ${ }^{1}{ }^{*}$ Ramchandra Bhatta $^{1}$, Keshav Raj Sigdel $^{2}$, and Rajendra Adhikari ${ }^{2}$ \\ ${ }^{1}$ Goldengate International College, Tribhuvan University, Kathmandu, Nepal and \\ ${ }^{2}$ Department of Physics, Kathmandu University, Dhulikhel, Kavre, Nepal,
}

(Dated: Received: date / Accepted: date)

\begin{abstract}
We conduct the first principle calculation based on Density Functional Theory (DFT) and Non-equilibrium Green Function (NEGF) approach using Generalized Gradient Approximation (GGA). We have investigated the structural, electronic, mechanical, and electronic transport properties of a one-dimensional monoatomic gold nanowire (AuNW). Plus, the influence of strain in geometrical and electronic properties also investigated. The nanowire is stable with sustainable cohesive energy of $-1.52 \mathrm{eV}$. The chemical stability is due to the partly ionic and covalent bonding between the gold atoms. The electronic band shows the metallic behavior of nanowire with a conductivity of $1 \mathrm{G}_{0}$. Furthermore, the structural and electronic properties of AuNW change significantly due to the external strain. The compressive strain decreases the bond length conversely, tensile strain increases the bond length from the equilibrium length. Similarly, the higher electronic energy states shift towards the Fermi level due to tensile strain. Furthermore, the mechanical strength of the NW is calculated by finding the tensile stiffness of the nanowire which found to be $30.31 \mathrm{eV} / \AA$ that is threefold less than carbyne. The transport property is studied by designing the electrode-device-electrode regime by eliminating the current quantization effect due to the contacts. We obtain the current-voltage $(I V)$ characteristics by varying the chemical potential in the electrode region. The current-voltage profile shows AuNW follows Ohomic current-voltage relation until current gets saturated. All these findings show that monoatomic AuNW is a potential candidate for electronic, nanomechanical, and transport applications.
\end{abstract}

\section{INTRODUCTION}

The finding of reducing the dimension from threedimensional (3D) graphite to two-dimensional(2D)[1] graphene suggests the further possibilities of lowdimensional materials. Further, several 2D materials like h-BN[2, 3], SiC[4], phosphorene[5], Transitional metal dichalcogenides[6], MXenes[7] have been studied theoretically and experimentally. Similarly, the Study of Low dimensional carbon allotropes zero-dimensional (0D) fullerenes[8],1D nanotubes[9] shows nobel results. Moreover, recent studies show that by reducing the size of the material, miniaturization of any technological device is possible[10, 11].The $1 \mathrm{D}$ nanowires are a very good example of the ultimate conductor miniaturization. These nanoscale systems reveal novel electronic, optical, mechanical, and transport properties as a result of the extreme carrier confinement. They are highly demanding for technological applications due to their typical properties like negative magnetoresistance, low thermal conductivity, quantum conductance, ferromagnetism, and quantum size effects[12].But upon reduction of the size down to nanometric scale, the design of new electronic devices and atomic-size systems has been a challenging work in the manufacturing process. Additionally, in a transport system, the junction impedance should be optimal to obtain the maximal electric power transfer, with no loss of information. Moreover, significant progress has been made in the fabrication techniques such

\footnotetext{
* shambhubhandari789@gmail.com
}

as mechanically controllable break junction (MCBJ)[13], transmission electron microscopy (TEM)[14], and scanning tunneling microscopy(STM)[15]. With current experimental techniques, it is now possible the synthesis of gold monoatomic chains as nano-contacts. The linear monoatomic gold nanowire $(\mathrm{AuNW})$ is one of the most widely studied $1 \mathrm{D}$ systems. It is expected to use in nano electro-mechanical systems(NEMS), very large-scale integration(VLSI) circuits, and ultra large scale integration(ULSI) circuits[16, 17]. Previously, Kondo et.al experimental studied the characterization of helical multishell gold NWs[18]. Similarly, The effects of free surface on the yielding of AuNWs were simulated by Diao et al[19]. The density functional theory calculation of gold cluster between linear and zigzag monoatomic AuNWs is performed by Xiao et al[20]. Recently, Jariwala et.al has reported linear temperature dependence calculations for the AuNW in different topologies using the First principle calculations[21].

Although there is a number of theoretical and experimental studies to investigate several properties of AuNWs. There are no specific study on the strain influenced electronic, structural properties. Additionally, the electronic transport properties of monoatomic AuNW have not been reported yet. Therefore, we have presented the first principal calculation to fulfill the study gap and present it systematically in this paper.

\section{COMPUTATIONAL DETAILS}

The structural, electronic, and mechanical properties calculations are executed by using the density functional theory as implemented in the Spanish Initiative for Elec- 
tronic Simulations with Thousands of Atoms(SIESTA) code[22, 23]. Transport properties are performed within the non-equilibrium Green's function theory as developed in the TranSIESTA[24] module. The pseudopotential is constructed by using Troullier Martins Method[25]. The generalized gradient approximation (GGA) function with Perdew-Burke-Ernzerhof(PBE) [26] is adopted to treat the effects of correlation and electronic exchange. In all the calculations, we used the Double Zeta Plus polarization (DZP) as basis sets. Monkhrost pack [27] scheme has been used for Brillouin zone integration by using $1 \times 1 \times 100$. An energy cutoff of 300 Ry is used for the expansion of the reciprocal space. The electron temperature was set equal to $300 \mathrm{~K}$ with a Fermi surface broadening. The atomic relaxation was achieved when the force reached the value of $0.002 \mathrm{eV} / \AA$ using the standard conjugate-gradient $(\mathrm{CG})$ technique. The convergence criteria for the energy of the self-consistent field is set to be $1.0 \times 10^{-5} \AA$. The vacuum gap of $25 \AA$ between adjacent chains in $\mathrm{x}$ and $\mathrm{y}$ directions has been used to prevent interactions between the adjacent unit cell. For transport properties calculation, we have determined the current through the scattering region sandwiched between two semi-infinite electrodes at a finite bias voltage using the Landauer-Bttiker formula[28].

$$
T(E, V)=\operatorname{tr}\left[\Gamma_{R}(E, V) G^{R}(E, V) \Gamma_{L}(E, V) G^{A}(E, V)\right]
$$

Where, $\Gamma$ denotes the coupling matrix for both side electrodes and $G^{A}$ is the Green's function of the device region. The electric current through the atomic scale device is given by integration of this transmission function, which is calculated by using the following relation.

$$
\mathrm{I}\left(V_{b}\right)=G_{0} \int_{\mu_{L}}^{\mu_{R}} T\left(E, V_{b}\right)\left[f\left(E-\mu_{L}\right)-f\left(E-\mu_{R}\right)\right] d E
$$

where $\mathrm{V}_{b}$ is the bias voltage applied to the electrode. $\mu_{L}$ and $\mu_{R}$ are the two electrochemical potentials on either side of electrodes. $\mathrm{T}\left(\mathrm{E}, \mathrm{V}_{b}\right)$ is the transmission coefficient at energy $\mathrm{E}$ and applied bias voltage $V_{b}$. Similarly, $\mathrm{f}(\mathrm{E})$ is the Fermi-Dirac distribution function. The electron transmission function and the current flow through the AuNW at finite bias have been calculated using the post-processing tool TBTrans which is included in the TranSIESTA module.

\section{RESULTS AND DISCUSSION}

\subsection{Equilibrium geometrical and electronic properties}

First, the fcc gold bulk (3D) structure is optimized by using the conjugate gradient (CG) approximation integrated into the SIESTA code. The optimized value of the lattice constant for the fcc 3D counterpart of the nanowire is found to be $4.10 \AA$ which is very close to the experimental measures. This ensures the accuracy of the pseudopotential. A unit cell consisting of four gold atoms is used for the electronic and mechanical properties calculation of AuNW. The optimized bond distance between gold atoms in the linear chain is $2.51 \AA$. Further, the cohesive energy of the nanowire is calculated by subtracting the energy of the isolated atom from the total energy of the system. The cohesive energy of the $\mathrm{AuNW}$ is $-1.52 \mathrm{eV} /$ atom. Furthermore, the cohesive energy and the nature of the chemical bond are closely correlated. The nature of the bond is observed by the Mulliken charge density analysis (FIG.1). There is a small distortion of the overlapped wave function at the center of $\mathrm{Au}$ atoms. That indicates that the gold atom possesses a partly ionic and partly covalent bond. These results are in good agreement with previous studies[21, 29].

(a)

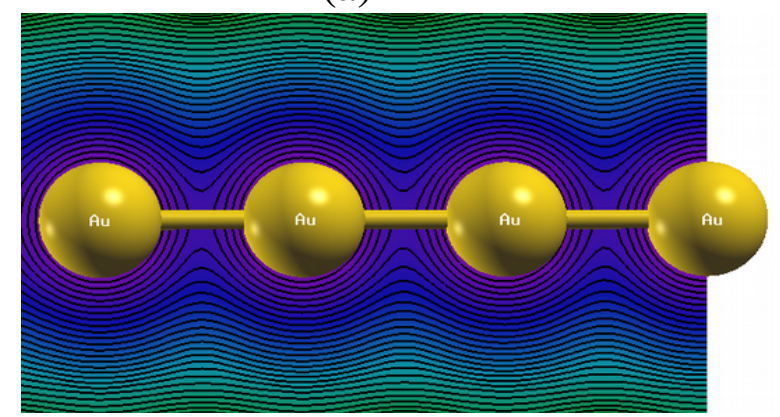

(b)

(c)
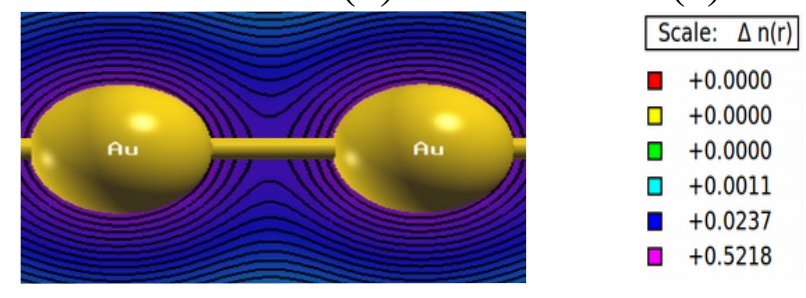

FIG. 1. (Color online) Total charge density contour plot of the unit cell of the AuNW (a) the zoomed view charge density between two gold atoms (c) charge density index index

Further, to get insight into the electronic properties of the nanowire, we execute the spin-independent electronic properties calculation. The band structure (FIG. 2(a)) shows that one band crosses the Fermi level that indicates the linear AuNW is purely metallic. According to the Landauer formulation, the amount of band which is crossing over the Fermi level represents the amount of quantum conductance or the number of conduction channel ' $G_{0}$ ' in units of $G_{0}=\frac{2 e^{2}}{h}[30]$ With this expression, the quantum conductance can be evaluated from the electronic band structures. The quantum conductance $G_{0}$ is associated with each state which is crossing over the Fermi level. Therefore, overall quantum conductance is given by $\mathrm{n}_{0}$, where, $\mathrm{n}$ indicates the number of channels crossing over the Fermi level. Since only one band is crossing the Fermi level, the quantum conductance is 
found to be $1 G_{0}$. Additionally, there are a large number of flat bands around the energy range in $-0.5 \mathrm{eV}$ and some flat band appears around $-2.1 \mathrm{eV}$ of the valence bands. These flat bands give rise to a variety of exotic manybody phenomena. This result is in very good agreement with the previous studies[31]. Further, the calculated projected density of states FIG. 2(b) depicts that the $5 d$ orbital is mainly contributing in the valence band region and conduction band minimum region closer to the Fermi level. But the contribution is dominated by the $6 \mathrm{~s}$ orbital in the higher energy region of the conduction band. It's worth mentioning that a similar orbital contribution is found fcc bulk counterpart[32]. (a)

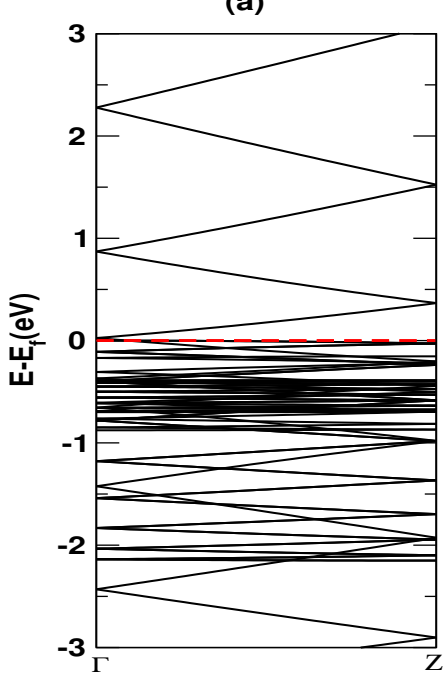

(b)

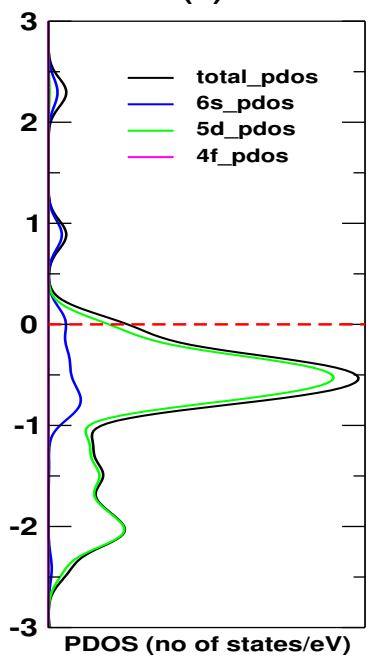

FIG. 2. (Color online) (a) Electronic bands (b) partial density of states (PDOS) of gold nanowire.The dashed vertical line represents the Fermi level set equal to $0 \mathrm{eV}$.

\subsection{Strain-induced geometric and electronic properties}

After realizing the properties of a strain-free AuNW, it's always crucial to explore the structural and electronic properties at a different range of strain loading conditions. The strain is applied by varying the lattice constants within the range of $-12 \%$ to $+12 \%$ at the step of $2 \%$. The applied strain can be expressed as $\varepsilon=\frac{\left(a-a_{0}\right)}{a_{0}}$ where $a_{0}$ and $a$ are equilibrium and strained lattice constant. The tensile and compressive strain is applied in AuNW to investigate the strain effect on the nanowire. The bond length between gold atoms linearly increases by tensile strain and decreases by the compressive strain (FIG. 3). The rate of change of bond length is almost the same for both types of strain. The change in bond length due to strain motivates to find its influence in electronic properties of the system. So, to get insight into electronic properties influenced by the application of ex- ternal strain(FIG. 4), we calculate the density of states (DOS) in within the elastic range of $12 \%$ to $+12 \%$ with the increment of $2 \%$. The maximum electronic state is shifted towards the Fermi level when strain is applied from $-12 \%$ to $12 \%$ altering the electronic properties.

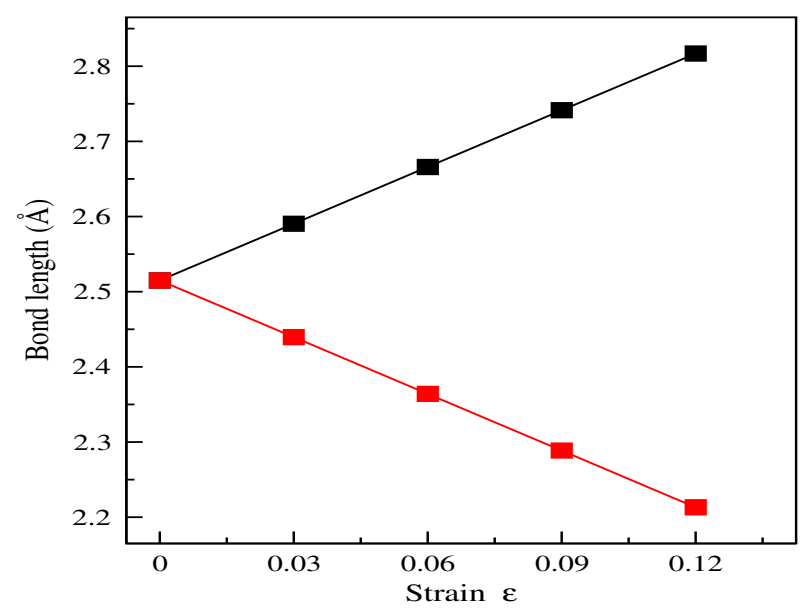

FIG. 3. (Color online) Effect of strain on the bondlength red(bond alteration due to compressive strain), black(bond alteration due to tensile strain).
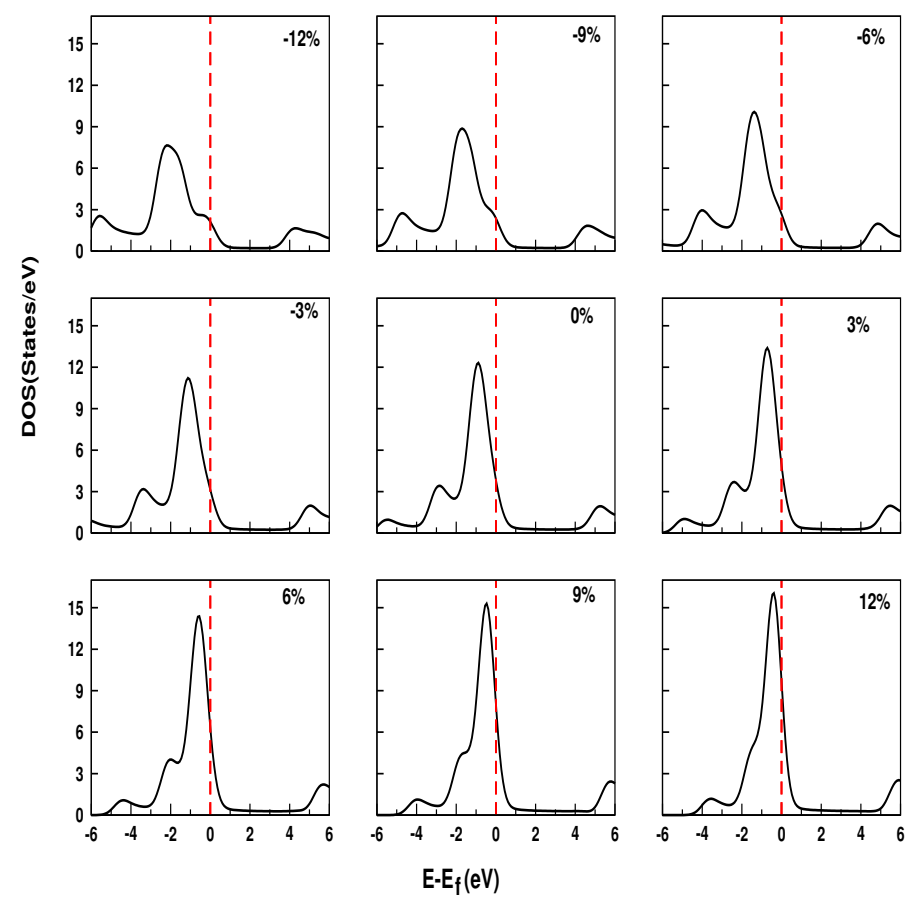

FIG. 4. (Color online) Variation of DOS with the applied external strain $(-12 \%$ to $+12 \%)$ around the elastic range. 


\subsection{Mechanical properties}

We investigate the mechanical strength of the nanowire by applying a uniaxial strain. The $1 \mathrm{D}$ ultrathin system is insufficient to find the tensile stiffness (C) in order to estimate the mechanical strength. We have followed the methodology of reference[33]. We have calculated the tensile stiffness of the nanowire by varying the lattice parameters within the range of $-2 \%$ to $+2 \%$ at the step of $0.5 \%$. The tensile stiffness is the most basic mechanical properties which are defined by $C=\frac{1}{a} \frac{\partial^{2} E}{\partial \varepsilon^{2}}$

Where $\mathrm{a}$ is the lattice constant, $\mathrm{E}$ is strain energy per unit cell. The strain energy is the difference between the energy of the strained and strain-free system. The tensile stiffness is calculated by fitting the relation above the equation. The fitted plot is shown in FIG. 5. The value of the tensile stiffness is found to be $31.31 \mathrm{eV} / \AA$.

It is worth mentioning that the linear chain of carbon atoms so-called "carbyne" shows the tensile stiffness $95.56 \mathrm{eV} / \AA[33]$. This confirms that the value of tensile stiffness of AuNW is three-fold less than the carbyne.

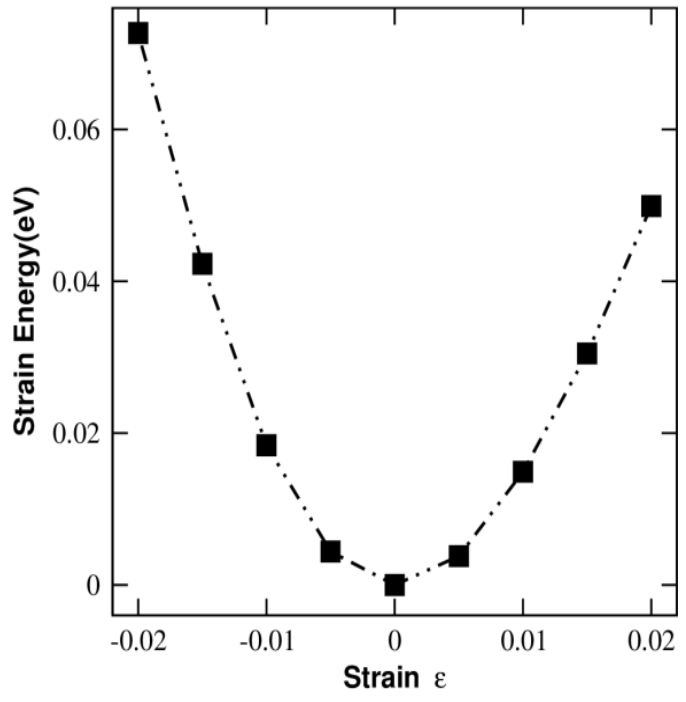

FIG. 5. (Color online) Strain versus strain energy fit.

\subsection{Transport Properties}

To perform the transport properties calculation of ultrathin AuNW, we use a total of $17 \mathrm{Au}$ atoms, out of which 4 atoms on either side are considered as electrodes and the remaining atoms in between have been chosen as scattering region or device region (FIG. 6). The size of the electrodes has been chosen to be the same in the left and right regions, eliminating current quantization effects due to contact electrodes. While designing the system for transport properties calculation, it is considered that the first electrodes size large enough to guarantee that orbitals within the unit cell only interact with one single nearest neighbor and the scattering region large enough to avoid interaction between the left electrode and right electrode to avoid the non-physical results. Electronic transmission function and the current flowing along the wire at constant bias conditions is calculated with the help of post-processing tool TBTrans of TranSIESTA.

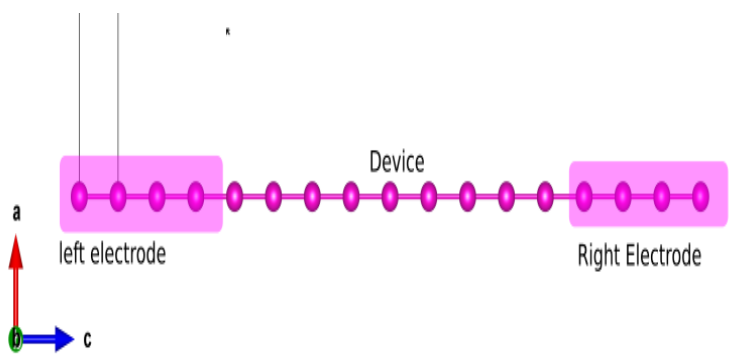

FIG. 6. (Color online) Ballstick representations of the equilibrium linear chain of $\mathrm{Au}$ with the three main components for transport analysis: semi-infinite left and right electrodes and scattering region (device).

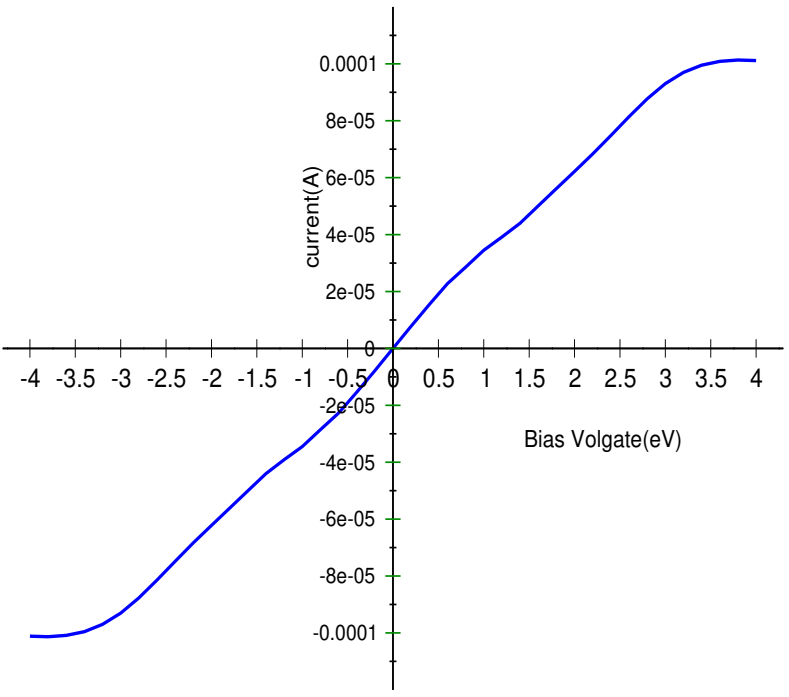

FIG. 7. (Color online) Current-voltage (IV) curves for the linear Au chain

The electronic band structure, the density of state, and transmission function (TF) for AuNW are shown in FIG. 8. The overall DOS and transmission function shape of the AuNW decays toward high energies as is typical for a one-dimensional system. Similarly, the current-voltage characteristic is shown in FIG. 7. The different bias voltage is applied to study the corresponding device currently. I-V characteristics are obtained by iterating the process for different bias voltage. The voltage is increased in steps of $0.1 \mathrm{~V}$ within the range of $-4 \mathrm{~V}$ to $+4 \mathrm{~V}$. The converged density matrix of the previous state is used as the initial guess for the next step as configured in the code itself. The IV plot is symmetric in both forward and 


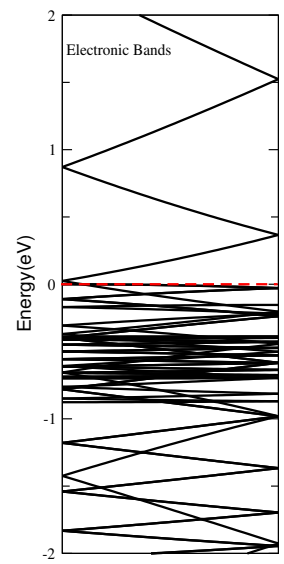

(a)
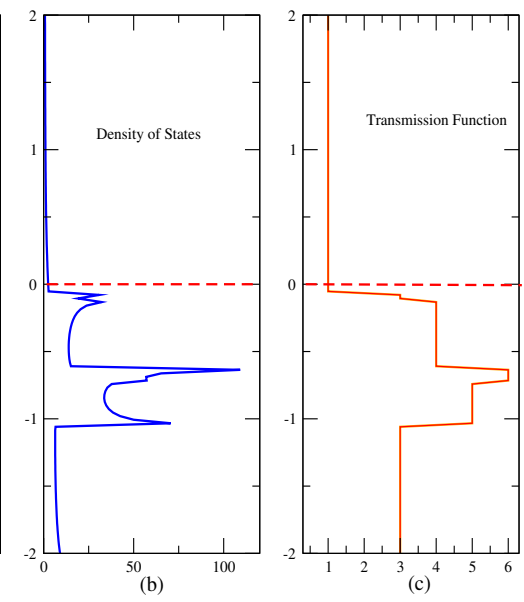

FIG. 8. (Color online) Electronic band structure, the density of state, and Transmission function for Au nanowire.

reverses bias. First, the current increases linearly with small fluctuation. Further, at a bias Voltage of $3.4 \mathrm{~V}$, the value of current saturates and constant current is observed. Similarly, the current-voltage relation is reported for the Aluminum chain in reference[34].

\section{CONCLUSIONS}

We have performed the first principle calculation with the DFT-NEGF approach to study the gold nanowire.
The stability of nanowire is confirmed by the sustainable value of cohesive energy. The linear AuNW is formed by a partly ionic and covalent bond between gold atoms. The electronic band structure has shown that AuNW is metallic with multiple flat bands in different energy ranges. Additionally, the structural and electronic properties are found to be influenced by the external strain. Plus, the tensile stiffness value has confirmed the mechanical strength and stability of the wire. The currentvoltage plot has shown the Ohomic behavior until the current gets saturated. The influence of external strain on transport properties is not covered in this work. The tuning of the current-voltage relation is also may be possible in the future. Hence, our finding confirms that monoatomic ultra-thin gold nanowire is a potential candidate in electronics, nanomechanical, and transport applications.

\section{ACKNOWLEDGMENT}

This research was supported in part through computational resources provided by the Kathmandu University Supercomputer Centre, which was established with equipment donated by CERN so. We are very grateful to the Supercomputer Center (Kathmandu University) for providing high performance computing facilities during the research work
[1] K. S. Novoselov, A. K. Geim, S. V. Morozov, D. Jiang, M. I. Katsnelson, I. Grigorieva, S. Dubonos, Firsov, and AA, nature 438, 197 (2005).

[2] Q. Peng, W. Ji, and S. De, Computational Materials Science 56, 11 (2012).

[3] L. Song, L. Ci, H. Lu, P. B. Sorokin, C. Jin, J. Ni, A. G. Kvashnin, D. G. Kvashnin, J. Lou, B. I. Yakobson, et al., Nano letters 10, 3209 (2010).

[4] Z. Shi, Z. Zhang, A. Kutana, and B. I. Yakobson, ACS nano 9, 9802 (2015).

[5] S. Das, M. Demarteau, and A. Roelofs, ACS nano 8, 11730 (2014).

[6] K. F. Mak, C. Lee, J. Hone, J. Shan, and T. F. Heinz, Physical review letters 105, 136805 (2010).

[7] J.-C. Lei, X. Zhang, and Z. Zhou, Frontiers of Physics 10, 276 (2015).

[8] R. Taylor and D. R. Walton, Nature 363, 685 (1993).

[9] S. Iijima, nature 354, 56 (1991).

[10] P. S. Peercy, Nature 406, 1023 (2000).

[11] Y. Huang, X. Duan, Y. Cui, L. J. Lauhon, K.-H. Kim, and C. M. Lieber, Science 294, 1313 (2001).

[12] L. Cademartiri and G. A. Ozin, Advanced Materials 21, 1013 (2009).

[13] M. A. Reed, C. Zhou, M. Deshpande, C. Muller, T. Burgin, L. Jones, and J. M. Tour, Annals of the New York Academy of Sciences 852, 133 (1998).
[14] H. Ohnishi, Y. Kondo, and K. Takayanagi, Nature 395, 780 (1998).

[15] A. Dhirani, P. Lin, P. Guyot-Sionnest, R. Zehner, L. Sita, S. Datta, W. Tian, S. Hang, R. Retfenberger, and J. H. C. Kubiak, J. Chem. Phys 106, 5249 (1997).

[16] A. N. Cleland and M. L. Roukes, Nature 392, 160 (1998).

[17] X. Duan, Y. Huang, Y. Cui, J. Wang, and C. M. Lieber, nature 409, 66 (2001).

[18] Y. Kondo and K. Takayanagi, Science 289, 606 (2000).

[19] K. Gall, J. Diao, and M. L. Dunn, Nano Letters 4, 2431 (2004).

[20] L. Xiao, B. Tollberg, X. Hu, and L. Wang, The Journal of chemical physics 124, 114309 (2006).

[21] P. Jariwala, S. K. Gupta, Y. Sonvane, Thakor, and PB, Superlattices and Microstructures 106, 206 (2017).

[22] J. M. Soler, E. Artacho, J. D. Gale, A. García, J. Junquera, P. Ordejón, and D. Sánchez-Portal, Journal of Physics: Condensed Matter 14, 2745 (2002).

[23] E. Artacho, D. Sánchez-Portal, P. Ordejón, A. Garcia, and J. M. Soler, physica status solidi (b) 215, 809 (1999).

[24] M. Brandbyge, J.-L. Mozos, P. Ordejón, J. Taylor, and K. Stokbro, Physical Review B 65, 165401 (2002).

[25] N. Troullier and J. L. Martins, Physical review B 43, 1993 (1991).

[26] J. P. Perdew, K. Burke, and M. Ernzerhof, Physical review letters 77, 3865 (1996). 
[27] H. J. Monkhorst and J. D. Pack, Physical review B 13, 5188 (1976).

[28] M. Büttiker, Y. Imry, R. Landauer, and S. Pinhas, Physical Review B 31, 6207 (1985).

[29] A. Kumar, A. K. Kaushal, and P. Ahluwalia, in AIP Conference Proceedings (American Institute of Physics, 2011), vol. 1349, pp. 1145-1146.

[30] J. Zhao, C. Buia, J. Han, and J. P. Lu, Nanotechnology 14, 501 (2003).

[31] A. Thakur, A. Kumar, and P. Ahluwalia, in AIP Conference Proceedings (American Institute of Physics, 2013), vol. 1512, pp. 190-191.

[32] L.-M. Yang, M. Dornfeld, T. Frauenheim, and E. Ganz, Physical Chemistry Chemical Physics 17, 26036 (2015).

[33] M. Liu, V. I. Artyukhov, H. Lee, F. Xu, and B. I. Yakobson, ACS nano 7, 10075 (2013).

[34] F. Sanchez-Ochoa, G. H. Cocoletzi, G. Canto, and N. Takeuchi, RSC advances 5, 91288 (2015). 